\title{
Effects of an individualized and time based training program on physical fitness and mood states in recreational endurance runners
}

\author{
Kuno Hottenrott ${ }^{1,2}$, Sebastian Ludyga ${ }^{2}$, Thomas Gronwald ${ }^{1,2}$, Stephan Schulze ${ }^{2}$ \\ ${ }^{1}$ Department of Sport Science, Martin-Luther-University Halle-Wittenberg, Halle, Germany \\ ${ }^{2}$ Institute of Performance Diagnostics and Health Promotion, Martin-Luther-University Halle-Wittenberg, Halle, Germany
}

\section{Email address:}

kuno.hottenrott@sport.uni-halle.de (K. Hottenrott)

\section{To cite this article:}

Kuno Hottenrott, Sebastian Ludyga, Thomas Gronwald, Stephan Schulze. Effects of an Individualized and Time Based Training Program on Physical Fitness and Mood States in Recreational Endurance Runners. American Journal of Sports Science.

Vol. 2, No. 5, 2014, pp. 131-137. doi: 10.11648/j.ajss.20140205.15

\begin{abstract}
Previous studies have shown that training guided individually by heart rate variability (HRV) elicits benefits on endurance performance. Meanwhile complex programs, which use HRV assessments to tailor exercise prescriptions to individual needs, are included in portable training computers. However, knowledge on health and fitness benefits of such training programs is still limited. Therefore, the aim of the present study was to investigate effects of HRV-based training included in a portable training computer vs general time-based training on aerobic fitness, half-marathon time as well as mood and mental states. Male $(n=10)$ and female endurance runners $(n=10)$ were recruited and randomly assigned to HRV-based (STAR) and predefined time-based training (TBT). At baseline and after 16 weeks of training an incremental exercise test with spirometry was performed to assess aerobic power. Additionally, mood and mental states were asked. After the training period all subjects took part in a half-marathon. Matched-pairs were selected to compare groups based on similar relative weekly energy expenditure during training. With no differences between groups, STAR and TBT reduced weight $(\mathrm{p}=0.029)$, body fat $(\mathrm{p}=0.002)$ as well as systolic and diastolic blood pressure $(\mathrm{p} \leq 0.001)$. Maximal oxygen uptake significantly increased from $43.0 \pm 8.3$ to $46.1 \pm 9.0 \mathrm{ml} \cdot \mathrm{min}^{-1} \mathrm{~kg}^{-1}(\mathrm{p} \leq 0.001)$ and $43.2 \pm 4.8$ to $46.7 \pm 4.7 \mathrm{ml}^{\mathrm{min}}{ }^{-1} \mathrm{~kg}^{-1}$ $(\mathrm{p} \leq 0.001)$ in STAR and TBT, respectively. Moreover, both groups improved maximal velocity (TBT: $0.81+0.40 \mathrm{~km} \mathrm{~h}^{-1} \mathrm{vs}$ STAR: $\left.0.74+0.39 \mathrm{kmh}^{-1} ; \mathrm{p} \leq 0.001\right)$ and completed the half-marathon with similar finish times $(01: 54: 35 \pm 00: 14: 02$ [hh:mm:ss] vs. 01:52:42 \pm 00:13:45 [hh:mm:ss]; $\mathrm{p}=0.789$ ). Regarding mood and mental states, no major changes were observed over the training period. In conclusion, both HRV- and time-based training elicited similar improvements in aerobic power and body composition. Consequently, HRV-dependent exercise prescriptions serve as a practical tool for day-to-day periodization of aerobic exercise.
\end{abstract}

Keywords: Heart Rate Variability, Aerobic Power, Mood States, Endurance Training, Recreational Athletes

\section{Introduction}

Improved aerobic fitness is associated with a variety of health benefits, such as reduced risk for all-cause mortality and cardiovascular diseases [1,2]. Therefore, recreational athletes seek to follow training programs that promise significant improvements in health-related outcomes. Considerable heterogeneity observed in the training response shows that changes in aerobic fitness do not only depend on training volume and intensity. Even after highly standardized training programs, the magnitude of improvements in maximal oxygen uptake has ranged from none to almost $40 \%[3,4,5]$. The underlying physiological mechanisms that cause individual differences in the responsiveness to endurance exercise are still not fully understood. To some extent individual differences in age, gender and training status partly explain the variance in the training response $[6,7,8]$. This might be due to a training stimulus that is not tailored to the individual needs of the athlete. Consequently, exercise scientists face the challenge to provide an effective training program, which allows the repetition of productive exercise without excessive accumulation of stress [9]. 
A key to initiate desirable changes in the cardiovascular system without compromising the recovery processes might be individualized training based on heart rate variability (HRV), because daily assessment of autonomic nervous system (ANS) activity could serve as an indicator of appropriate physiological condition for aerobic training [3]. Kiviniemi et al. [10] showed that a training guided individually by HRV (based on high frequency power) led to higher increases in maximal running velocity than a predefined training. Especially when there is an increase or no change in instantaneous beat-to-beat variability, a progression of exercise intensity promises enhancements of endurance performance in men and women [11]. The relation between the ANS activity and the responsiveness to specific training stimuli is further emphasized by Vesterinen et al. [12], who found that a high baseline HRV predicts desirable future exercise adaptations. Although HRV is usually assessed with ECG, the results of Gamelin et al. [13] also confirm valid measurements with heart rate monitors.

Due to the technical progress, some portable training computers now feature programs based on regular HRV assessments. This allows the adjustment of exercise prescriptions according to the individual needs of the user. Unfortunately, only a few studies on the effectiveness of such training programs are available. Ring-Dimitrou et al. [14] provide evidence that an exercise telemetry monitored training program elicits higher improvements of running performance at the aerobic threshold compared to a non-exercise telemetry monitored group. Recent findings by Hottenrott et al. [15] support the effectiveness of HRV-based training included in heart rate monitors, as recreational athletes increased their maximal oxygen uptake by $10.3 \%$ after 12 weeks of endurance exercise. However, more studies are necessary to evaluate health and performance benefits of individualized, self-administered training and time-based, predefined approaches.

The aim of the present study was to compare the effects of a standardized, time-based training plan with a training based on individual HRV measurements, which is included in a portable training computer, on aerobic performance, body composition and blood pressure. Additionally, the influence of the training approaches on mood and mental states were assessed. Following the intervention, half-marathon performance was compared between groups.

\section{Methods}

\subsection{Subjects}

Table 1. Subjects' characteristics at baseline.

\begin{tabular}{|c|c|c|c|c|c|}
\hline & \multicolumn{2}{|c|}{ STAR $(n=10)$} & \multicolumn{2}{|c|}{ TBT $(n=10)$} & \multirow{2}{*}{$\mathbf{p}$} \\
\hline & Mean & SD & Mean & SD & \\
\hline Age [y] & 42.3 & 7.2 & 40.7 & 9.9 & 0.236 \\
\hline Height $[\mathrm{cm}]$ & 172.2 & 8.1 & 170.2 & 10.8 & 0.279 \\
\hline Weight $[\mathrm{kg}]$ & 68.9 & 10.6 & 66.1 & 13.8 & 0.544 \\
\hline $\mathrm{BMI}\left[\mathrm{kg}^{-2}\right]$ & 23.1 & 1.9 & 22.7 & 2.7 & 0.401 \\
\hline Body Fat [\%] & 22.1 & 7.9 & 19.8 & 6.6 & 0.572 \\
\hline $\mathrm{VO}_{2 \operatorname{MAX}}\left[\mathrm{ml}^{-m^{-1}} \mathrm{~kg}^{-1}\right]$ & 43.0 & 8.3 & 43.2 & 4.8 & 0.786 \\
\hline
\end{tabular}

Male $(n=10)$ and female $(n=10)$ recreational endurance runners (Table 1) were recruited for a 16 week intervention period via advert in a local newspaper. Subjects had to fulfill the following inclusion criteria: age 30 to 50 years, non-smokers, weekly running distance of 10 to $20 \mathrm{~km}$ within the last 6 months, best $10 \mathrm{~km}$ time between 45 and $60 \mathrm{~min}$ and best half-marathon time between $2 \mathrm{~h}$ and $2 \mathrm{~h} 30 \mathrm{~min}$. Exclusion criteria were any cardiovascular, metabolic, neurological, pulmonary or orthopedic complications that could limit the performance of the exercises. Prior to the experimental trial recruited subjects underwent a medical check-up, including a personal anamnesis, ECG at rest and measurement of blood pressure. All eligible participants were given full details (except the purposes of the investigation) of the study protocol and any possible risks or discomforts associated with the experimental trial. Afterwards, written informed consent was obtained. The trial received approval by the local Ethics Committee and was in accordance with the Declaration of Helsinki.

\subsection{Study Design}

For an experimental trial with pre-post design recruited subjects were randomly assigned to either a HRV-based (STAR; $\mathrm{n}=10$ ) or time-based training program (TBT; $\mathrm{n}=10$ ). In contrast to TBT, training in STAR was regulated according to the individual response of the autonomic nervous system. At baseline and after 16 weeks of training an incremental exercise test with spirometry was performed to assess aerobic power. Additionally, mood and mental states were asked with the validated BFS scale [16] at baseline, 6 weeks and 16 weeks. One week after completing the post-test assessment all subjects took part in a half-marathon. Matched-pairs were selected to compare groups based on similar relative weekly energy expenditure during training.

\subsection{Exercise Testing}

Prior to exercise testing, anthropometric data were collected. Following the measurement of weight and body composition with a bio impedance device (Tanita, BC-545, Germany), subjects performed an incremental test with spirometry (Cortex, Metalyzer 3B, Germany) on a treadmill (h/p/cosmos, Pulsar 4.0, Germany). Therefore, the initial speed of $7.0 \mathrm{~km}^{-1}$ was increased by $1.5 \mathrm{~km}^{-1}$ per 3 min until volitional exhaustion. During the test, heart rate and respiratory parameters were recorded continuously. Lactate was measured with the enzymatic-amperometric method (Dr. Mueller, Super GL ambulance, Germany) in $10 \mu \mathrm{l}$ blood taken from an ear lobe after each stage. Collected Data was processed with the software WinLactat 3.1 (Mesics, Germany). Using the Dickhuth model [17], the individual anaerobic threshold was derived from the lactate-velocity curve.

\subsection{Training}

Subjects assigned to HRV-based training received a training computer (Polar Electro Oy, FT60, Finland) 
including STAR training program, which is compatible with polarpersonaltrainer.com via Polar FlowLink (Polar Electro Oy, Finland). This training program is based on personal information (weight, height, age, sex), fitness level, activity level as well as training goal and sets intensity and time targets in accordance with the response of the autonomic nervous system. Therefore, data collected from the exercise test were saved in the settings of the portable training computers. For the experimental trial the STAR group trained with the program's option "maximize performance". The STAR program provides three different intensity zones $\left(\mathrm{I}=60-70 \% \mathrm{HR}_{\mathrm{MAX}} ; \mathrm{II}=70-80 \% \mathrm{HR}_{\mathrm{MAX}} ; \mathrm{III}=80-90 \%\right.$ $\left.\mathrm{HR}_{\mathrm{MAX}}\right)$. When the target is set to "maximize performance", the program mainly prescribes exercise in zone II. STAR training is progressive, as weekly training targets are adjusted according to the current fitness level.

Subjects in TBT followed a well-known structured, time-based training plan designed to make non-elite runners finish a half-marathon in less than $2 \mathrm{~h}$ [18]. As subjects already had running experience, the first 4 weeks of the original plan were skipped. The training program required two short 30 to 45 min runs (Tuesday and Thursday) and one long run (Sunday) per week. As the training was progressive, time and distance of the endurance exercises were increased over the intervention period.

In both groups training data was recorded with a training computer and followed up online on www.polarpersonaltrainer.com. Within the intervention period the display of the training computer was covered in TBT to prevent subjects from controlling the heart rate.

\subsection{Statistics}

The statistical analysis was performed with SPSS 21.0 (IBM Statistics, USA) for Windows. Gaussian distribution of the data was tested with the Shapiro-Wilk-Test. By applying a one-way ANOVA, baseline values and half-marathon finish times were compared between groups. Repeated-measures analysis of variance (ANOVA) followed by Bonferroni as post-hoc test was used to assess possible training effects on performance and body composition. For primary outcomes time effects and interactions (time*group) were reported. As mood and mental state variables followed a non-normal distribution, the Wilcoxon test was applied to analyze differences between measurements within groups. For all statistical tests a $\mathrm{p}$ level of $\leq 0.05$ was considered statistically significant. The following variables were selected to investigate significant changes within and between the groups: maximal oxygen uptake $\left(\mathrm{VO}_{2 \mathrm{MAX}}\right)$, velocity at the individual anaerobic threshold $\left(\mathrm{V}_{\mathrm{IAT}}\right)$, maximal velocity $\left(\mathrm{V}_{\mathrm{MAX}}\right)$, blood lactate $(\mathrm{La})$, heart rate $(\mathrm{HR})$, weight, body fat, blood pressure, rating of mood states as well as half-marathon finish time.

\section{Results}

\subsection{Training}

Both TBT and STAR had similar relative energy expenditure of $35.2 \mathrm{kcal} \cdot(\mathrm{kg} \cdot \text { week })^{-1}$ during training. As displayed in figure 1 , mean exercise intensity and duration was different between groups. While runners in STAR had a higher training volume, their mean exercise intensity was lower in comparison to TBT. No differences between groups were observed in training adherence, as STAR and TBT achieved $87 \%$ and $90 \%$ of the weekly training targets, respectively.

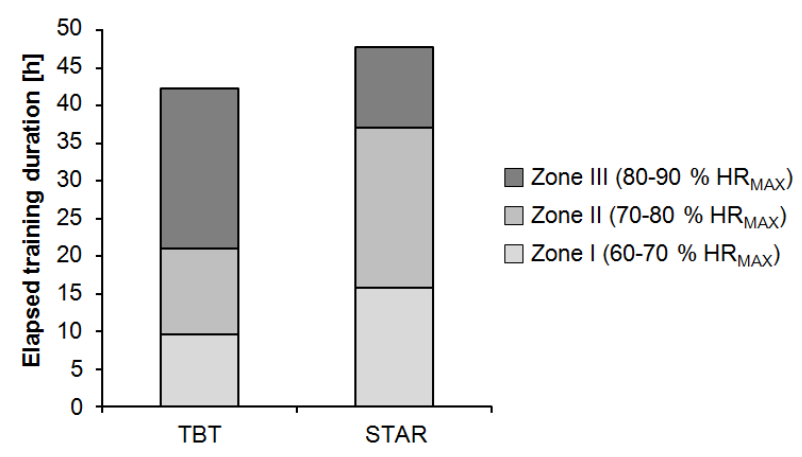

Figure 1. Training intensity and duration in TBT and STAR.

Table 2. Effect of HRV- (STAR; $n=5 \mathrm{f} / 5 \mathrm{~m})$ vs time-based training (TBT; $n=5 \mathrm{f} / 5 \mathrm{~m}$ ) on aerobic performance, blood lactate and heart rate.

\begin{tabular}{|c|c|c|c|c|c|c|}
\hline & \multirow{2}{*}{ Group } & \multicolumn{2}{|l|}{ Pre } & \multicolumn{2}{|l|}{ Post } & \multirow{2}{*}{$\begin{array}{l}\text { Pre vs Post } \\
\text { p }\end{array}$} \\
\hline & & Mean & SD & Mean & SD & \\
\hline \multirow{2}{*}{$\mathrm{V}_{\text {IAT }}\left[\mathrm{km} \cdot \mathrm{h}^{-1}\right]$} & STAR & 11.09 & 1.50 & 12.01 & 1.47 & $\leq 0.001$ \\
\hline & TBT & 11.75 & 0.97 & 12.55 & 0.99 & $\leq 0.001$ \\
\hline \multirow{2}{*}{$\mathrm{V}_{\text {MAX }}\left[\mathrm{km}^{-1}\right]$} & STAR & 13.87 & 1.51 & 14.54 & 1.53 & $\leq 0.001$ \\
\hline & TBT & 14.65 & 1.50 & 15.46 & 1.53 & $\leq 0.001$ \\
\hline \multirow{2}{*}{ 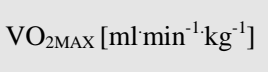 } & STAR & 43.00 & 8.25 & 46.12 & 9.02 & $\leq 0.001$ \\
\hline & TBT & 43.20 & 4.83 & 46.70 & 4.24 & $\leq 0.001$ \\
\hline \multirow{2}{*}{$\mathrm{La}_{\mathrm{MAX}}\left[\mathrm{mmol} \mathrm{l}^{-1}\right]$} & STAR & 8.43 & 2.45 & 8.39 & 2.71 & 0.864 \\
\hline & TBT & 8.59 & 2.40 & 9.24 & 2.29 & 0.111 \\
\hline \multirow{2}{*}{$\mathrm{HR}_{\mathrm{MAX}}\left[\min ^{-1}\right]$} & STAR & 185.40 & 9.95 & 184.44 & 11.46 & 0.578 \\
\hline & TBT & 185.40 & 12.20 & 185.20 & 12.67 & 0.883 \\
\hline
\end{tabular}

\subsection{Aerobic Performance and Half-Marathon}

Both groups increased maximal oxygen uptake, velocity at individual anaerobic threshold and maximal velocity $(\mathrm{p} \leq 0.001)$ over the intervention period (Table 2). As there were no significant time*group interactions $\left(\mathrm{VO}_{2 \mathrm{MAX}}\right.$ : $\left.\mathrm{p}=0,504, \mathrm{~V}_{\text {MAX }}: \mathrm{p}=0.739, \mathrm{~V}_{\text {IAT }}: \mathrm{p}=0,540\right)$, changes in selected performance parameters did not differ between 
STAR and TBT. Furthermore, maximal heart rate (STAR: $\mathrm{p}=0.578$; TBT: $\mathrm{p}=0.883$ ) and blood lactate concentration (STAR: $\mathrm{p}=0.864$; TBT: $\mathrm{p}=0.111$ ) was not altered by the exercise intervention. Following the training period, subjects in STAR and TBT completed the half-marathon with similar finish times $(01: 54: 35 \pm 00: 14: 02$ [hh:mm:ss] vs. 01:52:42 \pm 00:13:45 [hh:mm:ss]; $\mathrm{p}=0.789$ ).

\subsection{Mood and Mental States}

Over the training period mood and mental states in STAR were not altered in the assessed dimensions (Table 3). In comparison to baseline, excitation was significantly reduced after time-based training $(\mathrm{p}=0.047)$. Furthermore, anger decreased from the intermediate assessment at 6 weeks to post-test in TBT only $(p=0.034)$. Changes in frustration over the intervention period were significantly different between STAR and TBT $(\mathrm{p}=0.023)$. For all other domains of mood and mental states no interactions of time and group were observed.

Table 3. Subjects' mood and mental states at baseline. 6 weeks and 16 weeks of $H R V$ - $(S T A R, n=5 \mathrm{f} / 5 \mathrm{~m})$ and time-based training $(T B T ; n=5 \mathrm{f})$ $5 m)$.

\begin{tabular}{|c|c|c|c|c|c|c|c|}
\hline & \multirow{2}{*}{ Group } & \multicolumn{2}{|c|}{ Baseline } & \multicolumn{2}{|c|}{6 weeks } & \multicolumn{2}{|c|}{16 weeks } \\
\hline & & $\mathbf{M V}^{2}$ & SD & $\mathbf{M V}^{2}$ & SD & $\mathbf{M V}^{2}$ & SD \\
\hline \multirow{2}{*}{$\begin{array}{l}\text { Acti- } \\
\text { vation }^{1}\end{array}$} & STAR & 3.43 & 0.60 & 3.20 & 0.83 & 3.47 & 0.70 \\
\hline & TBT & 3.57 & 0.67 & 3.60 & 0.53 & 3.67 & 0.70 \\
\hline \multirow{2}{*}{ Anger $^{1}$} & STAR & 1.10 & 0.23 & 1.17 & 0.23 & 1.13 & 0.33 \\
\hline & TBT & 1.13 & 0.33 & 1.33 & 0.53 & $1.07^{\#}$ & 0.13 \\
\hline \multirow{2}{*}{$\begin{array}{l}\text { Positive } \\
\text { Mood }^{1}\end{array}$} & STAR & 3.53 & 0.70 & 3.37 & 0.97 & 3.63 & 0.70 \\
\hline & TBT & 3.63 & 0.90 & 4.07 & 0.70 & 3.67 & 0.63 \\
\hline \multirow{2}{*}{$\begin{array}{l}\text { Calm- } \\
\text { ness }^{1}\end{array}$} & STAR & 3.47 & 0.67 & 3.30 & 0.93 & 3.37 & 0.97 \\
\hline & TBT & 3.60 & 0.97 & 4.07 & 0.57 & 3.67 & 0.63 \\
\hline \multirow{2}{*}{$\begin{array}{l}\text { Exci- } \\
\text { tation }^{1}\end{array}$} & STAR & 1.67 & 0.63 & 1.60 & 0.63 & 1.77 & 0.73 \\
\hline & TBT & 1.77 & 0.57 & 1.63 & 0.47 & $1.47^{*}$ & 0.43 \\
\hline \multirow{2}{*}{$\begin{array}{l}\text { Frustra- } \\
\text { tion }^{1}\end{array}$} & STAR & 1.10 & 0.23 & 1.30 & 0.53 & 1.10 & 0.30 \\
\hline & TBT & 1.27 & 0.33 & 1.07 & 0.17 & 1.10 & 0.17 \\
\hline \multirow{2}{*}{ Fatigue $^{1}$} & STAR & 1.37 & 0.47 & 1.50 & 0.73 & 1.37 & 0.43 \\
\hline & TBT & 1.33 & 0.43 & 1.40 & 0.37 & 1.40 & 0.60 \\
\hline
\end{tabular}

${ }^{*} \mathrm{p} \leq 0.05$ in comparison to baseline; ${ }^{*} \mathrm{p} \leq 0.05$ in comparison to assessment at 6 weeks; ${ }^{1} 5$-point scale $\left(1=\right.$ not correct at all; $5=$ fully correct); ${ }^{2}$ mean values

\subsection{Body Composition and Blood Pressure}

Training significantly reduced body fat from $19.8 \pm 6.9$ to $18.5 \pm 6.6 \%$ and from $21.3 \pm 6.8$ to $19.7 \pm 6.6 \%(\mathrm{p} \leq 0.001)$ in TBT and STAR, respectively. Over the intervention period subjects' weight also decreased significantly in both groups (STAR: $-0.7 \pm 1.7 \mathrm{~kg}$; TBT: $-0.9 \pm 1.4 \mathrm{~kg}$; $\mathrm{p}=0.003$ ). Furthermore, the training interventions reduced blood pressure from $139.0 \pm 18.2 / 88.5 \pm 12.1$ to $132.7 \pm 17.2$ / $83.8 \pm 10.4 \mathrm{mmHg}$ in STAR (systolic: $\mathrm{p}=0.046$; diastolic: $\mathrm{p}=0.027)$ and from $138.5 \pm 15.9 / 86.5 \pm 10.2$ to $127.4 \pm 11.4$ / $79.9 \pm 7.2 \mathrm{mmHg}$ in TBT (systolic: $\mathrm{p}=0.009$; diastolic: $\mathrm{p}=0.028)$. As there were no interaction effects of time and group, the magnitude of reductions in body fat $(\mathrm{p}=0.859)$, weight $(\mathrm{p}=0.850)$ as well as systolic $(\mathrm{p}=0.550)$ and diastolic blood pressure $(\mathrm{p}=0.831)$ were not significantly different between STAR and TBT.

\section{Discussion}

\subsection{Aerobic Performance}

Based on a similar relative energy expenditure, both TBT and STAR elicited comparable improvements in aerobic power and maximal velocity after 16 weeks of training, although previous studies have shown that higher exercise intensities are more effective for improving cardiorespiratory fitness [19]. According to Midgley, McNaughton and Wilkinson [20], increased stroke volume due to structural changes of the myocard as well as improved capillarization and oxidative capacity of type II skeletal muscle fibers highly contribute to an enhancement of maximal oxygen uptake. Additionally, metabolic adaptations, which improve rates of fat oxidation and reduce the utilization of muscle glycogen at submaximal exercise ("glycogen sparing effect") benefit endurance performance [21] and might explain the rightward shift of the lactate velocity curve observed in the present trial.

Whereas the training plan of TBT was designed to make non-elite runners finish a half-marathon in less than $2 \mathrm{~h}$, the STAR program included in the portable training computers was originally not meant to prepare for running events. Nevertheless, subjects assigned to STAR completed the half-marathon with a time comparable to TBT. Previous studies have shown that the training response varies among subjects, because it is influenced by age, gender, stress, rest, prior training and baseline fitness [6,7]. Hence, the effectiveness of the STAR program might be explained by the fact that the training load was regulated in accordance with these factors. Especially the individual optimization of recovery periods was a significant strength of the HRV-based training as the recovery from exercise stress highly influences the training response [3]. In comparison to STAR runners in TBT followed a highly structured training plan, which did not pay attention to individual training needs. Nonetheless, this particular training method fulfilled its purpose, so that subjects were able to finish the marathon in the predefined time. To some extent, this might be due to the structured progression of training loads and a sufficient variability within the intervention period, as subjects performed both endurance runs and interval sessions.

In line with the present findings, Kiviniemi et al. [10] also found similar improvements of cardiorespiratory fitness after an individualized training guided by daily HRV measurements and a structured training. Some authors show that individualized training prescriptions elicit higher improvements in aerobic power [14,22] and foster motivation to subsequent physical activity [23]. To some extent, this heterogeneity among studies might be explained 
with differences in energy expenditure between the standardized and individualized training approaches. Additionally, the basis for tailoring exercise to individual needs varied among the trials. Especially training prescriptions based on regular assessments of the HRV might be recommended to recreational endurance athletes as A) HRV is a strong predictor of the exercise response [7] and B) prevents insufficient training as well as overtraining [24].

\subsection{Mood and Mental States}

In elite athletes assessments of mood and mental states are used to detect functional and non-functional overreaching [25]. Especially anger, vigor and fatigue are reliable indicators of overtraining [26] as those mood states correlate with training volume [27]. In TBT and STAR mood and mental states remained relatively stable over the intervention period, although there was a progression of exercise intensity and duration. The absence of major changes implies that both training programs provide a training load, which benefits aerobic performance in recreational runners while overstrain is avoided. However, exercise in TBT and STAR failed to elicit enhancements in positive mood, which are usually associated with endurance training [28]. This might be due to the fact that subjects in both groups already had a very positive mood at baseline and greater exercise benefits are expected among individuals reporting symptoms of depressed mood [29]. Furthermore, a positive mood and mental state might lead to a higher exercise adherence in the long-term. According to Williams et al. [30] the affective response to an acute moderate-intensity exercise stimulus predicts self-reported physical activity 6 and 12 months later. Consequently, the maintenance of a positive mood over the training period in STAR and TBT might be the key for performing exercise on a regular basis after the study.

\subsection{Body Composition and Blood Pressure}

Exercise leads to improved health benefits by specific biological mechanisms, such as improved body composition (e.g., through reduced abdominal adiposity and improved weight control), enhanced lipid lipoprotein profiles and reduced blood pressure [31,32]. In this respect, the decrease of body fat observed in this study is associated with a lower risk of type 2 diabetes [33] and all-cause mortality [1]. Additionally, HRV-based and time-based training were both efficient in reducing systolic and diastolic blood pressure. Especially the lowering of systolic blood pressure, which is a strong predictor of cardiovascular events, is related to a reduced risk of myocardial infarction and stroke [2]. Thus, training adaptations in both STAR and TBT were associated with relevant health benefits.

\section{Limitations}

As exercise intensity and duration were different between groups, the comparability of the training programs was limited. Therefore, the authors proceeded with pairs matched for the relative weekly energy expenditure to compare the effects of HRV-based and time-based training on aerobic performance. Unfortunately, this method led to a reduced sample size, which is known to affect the statistical results. Despite this limitation, the effects of the training programs on performance still reached the level of significance. Another methodological concern was the lack of half-marathon finish times at baseline. Nonetheless, the comparison of half-marathon performance after the training period was based on the assumption that finish times at baseline were not different between groups. This is supported by a similar initial aerobic fitness in STAR and TBT.

Regarding mood and mental state, time-based training led to marginal increases in anger and frustration. The lack of further changes might have been due to the limited number of the assessments. In future, more frequent measurements of mood and mental state over the training period could be useful to track differences between groups. Furthermore, the absence of group-related changes could be due to the training adherence in TBT. Subjects were expected to exercise on Tuesday, Thursday and Sunday. In some cases runners in TBT still followed the training prescriptions for time and distance, but rescheduled the training days. Consequently, they individualized training to some extent, although TBT was not meant for own adjustments.

\section{Conclusion}

Both HRV- and time-based training elicited improvements in aerobic power and body composition, which are associated with a risk reduction for cardiovascular diseases. Although the performance-enhancing effects were similar between the groups, especially the HRV-based training included in portable training computers might be recommended to recreational athletes, as it is more flexible, easy to follow and pays attention to individual needs. Progression of exercise intensity and duration did not affect anger and fatigue, so that HRV-based training prevents overstrain by controlling regeneration processes continuously. Consequently, HRV-dependent exercise prescriptions can serve as a practical tool for day-to-day periodization of aerobic training.

\section{References}

[1] Bigaard, J., Frederiksen, K., Tjonneland, A., Thomsen, B.L., Overvad, K., Heitmann, B.L. \& Sorensen, T.I.A. (2004). Body fat and fat-free mass and all-cause mortality. Obesity Res, 12 (7), 1042-1049.

[2] Psaty, B.M., Furberg, C.D., Kuller, L.H., Cushman, M., Savage, P.J., Levine, D., O'Leary, D.H., Bryan, R.N., Anderson, M. \& Lumley, T. (2001). Association between blood pressure level and the risk of myocardial infarction, stroke, and total mortality. The cardiovascular health study. Arch Intern Med, 161 (9), 1183-1192. 
[3] Hautala, A.J., Kiviniemi, A.M. \& Tulppo, M.P. (2009) Individual responses to aerobic exercise: the role of the autonomic nervous system. Neurosci Biobehav Rev, 33 (2), 107-115.

[4] Vollaard, N.B., Constantin-Teodosiu, D., Fredriksson, K., Rooyackers, O., Jansson, E., Greenhaff, P.L., Timmons, J.A. \& Sundberg, C.J. (2009). Systematic analysis of adaptations in aerobic capacity and submaximal energy metabolism provides a unique insight into determinants of human aerobic performance. J Appl Physiol,106 (5), 1479-86.

[5] Buchheit, M., Chivot, A., Parouty, J., Mercier, D., Al Haddad, H., Laursen, P.B. \& Ahmaidi, S. (2010). Monitoring endurance running performance using cardiac parasympathetic function. Eur $J$ Appl Physiol, 108 (6),1153-67.

[6] Bouchard, C. \& Rankinen, T. (2001). Individual differences in response to regular physical activity. Med Sci Sports Exerc, $33,446-$ S451.

[7] Hautala, A.J., Mäkikallio, T.H., Kiviniemi, A., Laukkanen, R.T., Nissilä, S., Huikuri, H.V. \& Tulppo, M.P. (2003). Cardiovascular autonomic function correlates with the response to aerobic training in healthy sedentary subjects. $A m$ J Physiol Heart Circ Physiol, 285 (4), H1747-1752.

[8] Hautala, A.J., Kiviniemi, A.M., Makikallio, T.H., Kinnunen, H., Nissila, S., Huikuri, H.V. \& Tulppo, M.P. (2006). Individual differences in the responses to endurance and resistance training. Eur J Appl Physiol, 96, 535-542.

[9] Friden, J., Lieber, R.I., Hargreaves, M. \& Urhausen, A. (2003). Recovery after training-Inflammation, Metabolism, Tissue Repair and Overtraining, Sports Medicine. Malden: Blackwell Science, pp. 189-200.

[10] Kiviniemi, A.M., Hautala, A.J., Kinnunen, H. \& Tulppo, M.P (2007). Endurance training guided individually by daily heart rate variability measurements. Eur J Appl Physiol, 101 (6), 743-751.

[11] Kiviniemi, A. M., Hautala, A. J., Kinnunen, H., Nissila, J., Virtanen, P., Karjalainen, J. \& Tulppo, M. P. (2010). Daily exercise prescription on the basis of HR variability among men and women. Med Sci Sports Exerc, 42 (7), 1355-1363.

[12] Vesterinen, V., Häkkinen, K., Hynynen, E., Mikkola, J., Hokka, L. \& Nummela, A. (2013). Heart rate variability in prediction of individual adaptation to endurance training in recreational endurance runners. Scand J Med Sci Sports, 23 (2), 171-180.

[13] Gamelin, F.X., Berthoin, S. \& Bosquet, L. (2006). Validity of the polar S810 heart rate monitor to measure R-R intervals at rest. Med Sci Sports Exerc, 38(5), 887-893.

[14] Ring-Dimitriou, S., von Duvillard, S.P., Stadlmann, M., Kinnunen, H., Drachta, O., Müller, E., Laukkanen, R., Hamra, J., Weeks, S. \& Peak, K. (2008). Changes in physical fitness in moderately fit adults with and without the use of exercise telemetry monitors. Eur J Appl Physiol, 102, 505-513.

[15] Hottenrott, K., Ludyga, S. \& Schulze, S. (2013) Evaluierung von unterschiedlichen Trainingsmodellen bei berufstätigen Freizeitläufern. Sportwiss, 43 (3), 157-165.

[16] Abele, A. \& Brehm, W. (1986). Zur Konzeptualisierung und Messung von Befindlichkeit. Die Entwicklung der Befindlichkeitsskalen (BFS). Diagnostica, 32, 209-228.
[17] Dickhuth, H.-H., Huonker, M., Münzel, T., Drexler, H., Berg, A. \& Keul, J. (1991). Individual anaerobic threshold for evaluation of competitive athletes and patients with left ventricular dysfunction. In N. Bachl, T.E. Graham \& H. Löllgen (Hrsg.), Advances in ergometry (pp. 173-179). Berlin, Heidelberg: Springer Verlag.

[18] Galloway, J. (2006). Half-marathon: You Can Do It. Meyer \& Meyer Sport, Oxford, 64.

[19] Gormley, S.E., Swain, D.P., High, R., Spina, R.J., Dowling, E.A., Kotipalli, U.S. \& Gandrakota, R. (2008). Effect of Intensity of Aerobic Training on VO2max. Med Sci Sports Exerc, 40, 1336-1343.

[20] Midgley, A.W., McNaughton, L.R., Wilkinson, M. (2012). Is there an Optimal Training Intensity for Enhancing the Maximal Oxygen Uptake of Distance Runners? Sports Medicine, 36, 117-132.

[21] Yeo, W.K., Carey, A.L., Burke, L., Spriet, L.L. \& Hawley, J.A. (2011). Fat adaptation in well-trained athletes: effects on cell metabolism. Appl Physiol, Nutr Metabol, 36 , 12-22.

[22] Fabre, C., Massé-Biron, J., Ahmaidi, S., Adam, B. \& Préfaut, C. (1997). Effectiveness of individualized aerobic training at the ventilatory threshold in the elderly. J Gerontol A Biol Sci Med Sci, 52 (5), 260-266.

[23] Lafortuna, C.L., Resnik, M., Galvani, C. \& Sartorio, A. (2003). Effects of non-specific vs individualized exercise training protocols on aerobic, anaerobic and strength performance in severely obese subjects during a short-term body mass reduction program. J Endocrinol Invest, 26 (3),197-205.

[24] Mourot, L., Bouhaddi, M., Perrey, S., Cappelle, S., Henriet, M.T., Wolf, J.P., Rouillon, J.D. \& Regnard J. (2004). Decrease in heart rate variability with overtraining: assessment by the Poincaré plot analysis. Clin Physiol Funct Imaging, 24 (1), 10-18.

[25] Rietjens, G.J.W.M., Kuipers, H., Adam, J.J., Saris, W.H.M., van Breda, E., van Hamont, D. \& Keizer, H.A. (2005). Physiological, biochemical and psychological markers of strenuous training-induced fatigue. Int J Sports Med, 26 (1), $16-26$.

[26] Grant, C.C., Janse Van Rensburg, D.C., Collins, R., Wood, P.S. \& Du Toit, P.J. (2012). Investigation of the Profile of Mood State (POMS) questionnaire as an indicator of Overtraining Syndrome (OTS) in a group of endurance athletes. AJPHERD, 23-32.

[27] Pierce, E.F. (2002). Relationship between training volume and mood states in competitive swimmers during a 24-week season. Percept Mot Skills, 94 (3), 1009-1012.

[28] Peluso, M.A.M. \& de Andrade, L.H.S.G. (2005). Physical activity and mental health: The association between exercise and mood. Clinics, 60 (1), 61-70.

[29] Lane, A.M. \& Lovejoy, D.J. (2001). The effects of exercise on mood changes: the moderating effect of depressed mood. J Sports Med Phys Fitness, 41 (4), 539-545.

[30] Williams, D.M., Dunsiger, S., Ciccolo, J.T., Lewis, B.A., Albrecht, A.E. \& Marcus, B.H. (2008). Acute Affective Response to a Moderate-intensity Exercise Stimulus Predicts Physical Activity Participation 6 and 12 Months Later. Psychol Sport Exerc, 9 (3), 231-245. 
[31] Maiorana, A., O'Driscoll, G. \& Taylor, R. (2003). Exercise and the nitric oxide vasodilator system. Sports Med, 33, 1013-1035.

[32] Warburton, D.E., Gledhill, N. \& Quinney, A. (2001). The effects of changes in musculoskeletal fitness on health. Can J Appl Physiol, 26, 161-216.
[33] Meisinger, C., Döring, A., Thorand, B., Heier, M. \& Löwel, H. (2006). Body fat distribution and risk of type 2 diabetes in the general population: are there differences between men and women? The MONICA/KORA Augsburg Cohort Study. Am J Clin Nutr, 84 (3), 483-489. 